University of Nebraska - Lincoln

DigitalCommons@University of Nebraska - Lincoln

Comparison of a novel passive sampler to standard water-column sampling for organic contaminants associated with wastewater effluents entering a New Jersey stream

D. A. Alvarez

U.S. Geological Survey, dalvarez@usgs.gov

P. E. Stackelberg

U.S. Geological Survey

J. D. Petty

U.S. Geological Survey

J. N. Huckins

U.S. Geological Survey

E. T. Furlong

U.S. Geological Survey

See next page for additional authors

Follow this and additional works at: https://digitalcommons.unl.edu/usgsstaffpub

Part of the Earth Sciences Commons

Alvarez, D. A.; Stackelberg, P. E.; Petty, J. D.; Huckins, J. N.; Furlong, E. T.; Zaugg, S. D.; and Meyer, M. T., "Comparison of a novel passive sampler to standard water-column sampling for organic contaminants associated with wastewater effluents entering a New Jersey stream" (2005). USGS Staff -- Published Research. 363.

https://digitalcommons.unl.edu/usgsstaffpub/363

This Article is brought to you for free and open access by the US Geological Survey at DigitalCommons@University of Nebraska - Lincoln. It has been accepted for inclusion in USGS Staff -- Published Research by an authorized administrator of DigitalCommons@University of Nebraska - Lincoln. 


\section{Authors}

D. A. Alvarez, P. E. Stackelberg, J. D. Petty, J. N. Huckins, E. T. Furlong, S. D. Zaugg, and M. T. Meyer 


\title{
Comparison of a novel passive sampler to standard water-column sampling for organic contaminants associated with wastewater effluents entering a New Jersey stream
}

\author{
D.A. Alvarez ${ }^{\text {a,* }}$, P.E. Stackelberg ${ }^{\text {b }}$, J.D. Petty ${ }^{\text {c }}$, J.N. Huckins ${ }^{\text {a }}$, \\ E.T. Furlong ${ }^{\text {d }, ~ S . D . ~ Z a u g g ~}{ }^{\text {d }}$, M.T. Meyer ${ }^{\text {e }}$ \\ ${ }^{a}$ US Geological Survey, CERC, 4200 New Haven Road, Columbia, MO 65201, USA \\ ${ }^{\mathrm{b}}$ US Geological Survey, West Trenton, NJ 08628, USA \\ ${ }^{\text {c } U S ~ G e o l o g i c a l ~ S u r v e y ~ B R D ~ C e n t r a l ~ R e g i o n ~ O f f i c e, ~ C o l u m b i a, ~ M O ~ 65211, ~ U S A ~}$ \\ ${ }^{\mathrm{d}}$ US Geological Survey, Denver Federal Center, Denver, CO 80225, USA \\ ${ }^{\mathrm{e}}$ US Geological Survey, Lawrence, KS 66049, USA
}

Received 3 September 2004; received in revised form 21 February 2005; accepted 11 March 2005

Available online 27 April 2005

\begin{abstract}
Four water samples collected using standard depth and width water-column sampling methodology were compared to an innovative passive, in situ, sampler (the polar organic chemical integrative sampler or POCIS) for the detection of 96 organic wastewater-related contaminants (OWCs) in a stream that receives agricultural, municipal, and industrial wastewaters. Thirty-two OWCs were identified in POCIS extracts whereas 9-24 were identified in individual water-column samples demonstrating the utility of POCIS for identifying contaminants whose occurrence are transient or whose concentrations are below routine analytical detection limits. Overall, 10 OWCs were identified exclusively in the POCIS extracts and only six solely identified in the water-column samples, however, repetitive water samples taken using the standard method during the POCIS deployment period required multiple trips to the sampling site and an increased number of samples to store, process, and analyze. Due to the greater number of OWCs detected in the POCIS extracts as compared to individual water-column samples, the ease of performing a single deployment as compared to collecting and processing multiple water samples, the greater mass of chemical residues sequestered, and the ability to detect chemicals which dissipate quickly, the passive sampling technique offers an efficient and effective alternative for detecting OWCs in our waterways for wastewater contaminants.
\end{abstract}

Published by Elsevier Ltd.

Keywords: POCIS; Pharmaceuticals; Wastewater; Agricultural chemicals; Hydrophilic contaminants

\footnotetext{
* Corresponding author. Tel.: +1 573441 2970; fax: +1 573 8761896.

E-mail address: dalvarez@usgs.gov (D.A. Alvarez).
}

\section{Introduction}

The demand on freshwater to sustain the needs of the growing population is of worldwide concern. Often this water is used, treated, and released for reuse by other communities. The anthropogenic contaminants present 
in this water may include complex mixtures of pesticides, prescription and nonprescription drugs, personal care and common consumer products, industrial and domestic-use materials and degradation products of these compounds. The fate of such contaminants in wastewater treatment facilities is largely unknown, however, the limited data available suggests that many of these chemicals survive treatment and some are returned to their biologically active form via deconjugation of metabolites (Desbrow et al., 1998; Halling-Sørensen et al., 1998; Daughton and Ternes, 1999). Of greater concern is a study showing that many of these chemicals also survive treatment in drinking water plants and are present in finished waters (Stackelberg et al., 2004).

Traditional monitoring programs consist of collecting samples of one or more liters of environmental water or wastewater at specific points of time, performing sample enrichment in the laboratory (i.e., liquid-liquid extraction, solid-phase extraction, etc.), sample cleanup to remove potential interferences which may consist of size-exclusion chromatography, sorptive chromatography cleanup and/or fractionation, followed by instrumental analysis by gas chromatography (GC), gas chromatography/mass spectrometry (GC/MS), or high performance liquid chromatography (HPLC) (Barceló and Hennion, 1997; Petty et al., 2000; Kolpin et al., 2002; Hilton and Thomas, 2003). Advances in technology have led to the development of liquid chromatography/mass spectrometry (LC/MS) systems which enables scientists to expand their monitoring and assessment capabilities to include the more nonvolatile and water soluble organic contaminants (Kolpin et al., 2002; Richardson, 2002; Hilton and Thomas, 2003).

The traditional water-column sampling methodologies have many shortcomings. The volume of water sampled may be insufficient to satisfy the detection limit requirement of commonly used analytical methods. Traditional water samples represent only those contaminants present at the time of sampling. Episodic events such as spills or stormwater runoff are often missed as the contaminants can dissipate prior to the next sampling interval. Transient occurrence of selected contaminants in wastewater may result in temporal changes in the chemical quality of effluent discharged to neighboring streams. Repetitive sampling to accommodate episodic events and/or transient occurrence can be physically, logistically, and financially difficult, especially in remote areas. Without sufficient repetitive sampling, it may be impossible to formulate estimates on the time-weighted average (TWA) concentrations of the contaminants of interest. Determination of TWA concentrations is a fundamental part of an ecological risk assessment process for chemical stressors (Huckins et al., 2002a).

Passive samplers offer an attractive alternative to traditional sampling methods (Huckins et al., 1990; Lebo et al., 1995; Gustavson and Harkin, 2000; Petty et al., 2000; Huckins et al., 2002a; Alvarez et al., 2004; Petty et al., 2004). The success of personal dosimeters, or passive monitors, in determining TWA exposure concentrations of organic vapors in occupational environments (Fowler, 1982; ACGIH, 1990) has contributed to the application of the same principle to dissolved organic contaminants in aquatic environments. The use of integrative passive samplers enables estimates of TWA concentrations of contaminants of interest, permits sequestration of residues from episodic events commonly not detected with grab sampling, is not limited to constant water conditions, and allows the concentration of ultra-trace, yet toxicologically relevant, contaminant mixtures over extended periods of time (Huckins et al., 2002a; Alvarez et al., 2004; Petty et al., 2004).

In this study, we compared the polar organic chemical integrative sampler (POCIS) to standard water-column sampling methodologies for the detection of 96 organic wastewater-related contaminants (OWCs) including pesticides, prescription and nonprescription drugs, personal care and common consumer products, fragrances, fire retardants, plasticizers, and other components of industrial, domestic, and agricultural wastewaters and their select degradation products (Tables 1-4). The POCIS consists of a sequestration medium enclosed within a hydrophilic microporous polyethersulfone membrane (Fig. 1) for the integrative sampling of polar organic chemicals (Petty et al., 2002; Alvarez et al., 2004; Petty et al., 2004). The sampler is versatile as the sequestrering medium, composed of a solid-phase sorbent or mixture of sorbents, can be changed to target specific chemicals or chemical classes. It is common to have POCIS of several different configuration deployed together to maximize the information obtained. There are two configurations of POCIS that are typically used. One is a generic system which is useful for general hydrophilic organic contaminant purposes and the other is for pharmaceutical sampling. The generic configuration contains the triphasic sorbent admixture of Isolute ENV+ polystyrene divinylbenzene (Argonaut Technologies, Redwood City, CA, USA) and Ambersorb 1500 carbon (Rohm and Haas, Philadelphia, PA, USA) dispersed on S-X3 Biobeads (200-400 mesh, Bio-Rad, Hercules, CA, USA). This mixture exhibits excellent trapping and recovery of many pesticides, natural and synthetic hormones, and other wastewater-related contaminants (Steur-Lauridsen, 2003; Alvarez et al., 2004). The pharmaceutical configuration uses the Oasis HLB sorbent (Waters, Milford, MA, USA) for sequestering the chemicals. This configuration was necessary as many pharmaceuticals, with their multiple functional groups, had a tendency to strongly bind to the carbonaceous component of the sorbent admixture. The membrane acts as a semipermeable membrane, allowing 
Table 1

Prescription and nonprescription pharmaceuticals detected at Site 1

\begin{tabular}{|c|c|c|c|c|c|c|}
\hline Chemical (method) & Use & $\begin{array}{l}\text { Water } \\
\text { Rep. \#1 } \\
\text { ng/1 }\end{array}$ & $\begin{array}{l}\text { Water } \\
\text { Rep. \#2 } \\
\text { ng/1 }\end{array}$ & $\begin{array}{l}\text { Water } \\
\text { Rep. \#3 } \\
\text { ng/1 }\end{array}$ & $\begin{array}{l}\text { Water } \\
\text { Rep. \#4 } \\
\text { ng/1 }\end{array}$ & $\begin{array}{l}\text { POCIS } \\
\text { ng/POCIS }\end{array}$ \\
\hline Acetaminophen (1) & Analgesic & 7.1 & $<9.0$ & 14 & $<9.0$ & 4.1 \\
\hline Albuterol (salbutamol) (1) & Antiasthmatic & $<29$ & $<29$ & $<29$ & $<29$ & $<29$ \\
\hline Carbadox (2) & Antibiotic & $<50$ & $<50$ & $<50$ & $<50$ & $<50$ \\
\hline Carbamazepine (1) & Anticonvulsive & 73 & 95 & 130 & 44 & 240 \\
\hline Chlortetracycline (2) & Antibiotic & $<20$ & $<20$ & $<20$ & $<20$ & $<20$ \\
\hline Cimetidine (1) & Antacid & $<7$ & $<7$ & $<7$ & $<7$ & $<7$ \\
\hline Ciprofloxacin (2) & Antibiotic & $<10$ & $<10$ & $<10$ & $<10$ & $<10$ \\
\hline Codeine (1) & Analgesic & $<240$ & $<240$ & $<240$ & $<240$ & $<240$ \\
\hline Dehydronifedipine (1) & Antianginal & 8.2 & 14 & 18 & 5.1 & 25 \\
\hline Demeclocycline (2) & Antibiotic & $<20$ & $<20$ & $<20$ & $<20$ & $<20$ \\
\hline Diltiazem (1) & Antihypertensive & 15 & $<12$ & $<12$ & $<12$ & $<12$ \\
\hline Diphenhydramine (1) & Antihistaminic & 5.8 & 5.4 & $<15$ & $<15$ & 31 \\
\hline Doxycycline (2) & Antibiotic & $<50$ & $<50$ & $<50$ & $<50$ & $<50$ \\
\hline Enrofloxacin (2) & Antibiotic & $<10$ & $<10$ & $<10$ & $<10$ & $<10$ \\
\hline Erythromycin- $\mathrm{H}_{2} \mathrm{O}(2)$ & Erythromycin metabolite & $<20$ & $<20$ & $<20$ & $<20$ & $<20$ \\
\hline Fluoxetine (1) & Antidepressant & $<18$ & $<18$ & $<18$ & $<18$ & $<18$ \\
\hline Furosemide (1) & Diuretic & $<39$ & $<39$ & $<39$ & $<39$ & $<39$ \\
\hline Gemfibrozil (1) & Antihyperlipidemic & $<15$ & $<15$ & $<15$ & $<15$ & $<15$ \\
\hline Ibuprofen (1) & Analgesic & $<18$ & $<18$ & $<18$ & $<18$ & $<18$ \\
\hline Lincomycin (2) & Antibiotic & $<10$ & $<10$ & $<10$ & $<10$ & $<10$ \\
\hline Methotrexate (2) & Antirheumatic & $<20$ & $<20$ & $<20$ & $<20$ & $<20$ \\
\hline Miconazole (1) & Antifungal & $<18$ & $<18$ & $<18$ & $<18$ & $<18$ \\
\hline Minocycline (2) & Antibiotic & $<20$ & $<20$ & $<20$ & $<20$ & $<20$ \\
\hline Norfloxacin (2) & Antibiotic & $<10$ & $<10$ & $<10$ & $<10$ & $<10$ \\
\hline Oxytetracycline (2) & Antibiotic & $<50$ & $<50$ & $<50$ & $<50$ & $<50$ \\
\hline Ranitidine (1) & Antacid & $<10$ & $<10$ & $<10$ & $<10$ & $<10$ \\
\hline Roxithromycin (2) & Antibiotic & $<10$ & $<10$ & $<10$ & $<10$ & $<10$ \\
\hline Sarafloxacin (2) & Antibiotic & $<10$ & $<10$ & $<10$ & $<10$ & $<10$ \\
\hline Sulfachlorpyradazine (2) & Antibiotic & $<50$ & $<50$ & $<50$ & $<50$ & $<50$ \\
\hline Sulfadimethoxine (2) & Antibiotic & $<10$ & $<10$ & $<10$ & $<10$ & $<10$ \\
\hline Sulfamerazine (2) & Antibiotic & $<20$ & $<20$ & $<20$ & $<20$ & $<20$ \\
\hline Sulfamethazine (2) & Antibiotic & $<10$ & $<10$ & $<10$ & $<10$ & $<10$ \\
\hline Sulfamethoxazole (1) & Antibiotic & 34 & 22 & 22 & $<23$ & 9.5 \\
\hline Sulfamethoxazole (2) & Antibiotic & $<50$ & $<50$ & $<50$ & $<50$ & $<50$ \\
\hline Sulfathiazole (2) & Antibiotic & $<50$ & $<50$ & $<50$ & $<50$ & $<50$ \\
\hline Tetracycline (2) & Antibiotic & $<20$ & $<20$ & $<20$ & $<20$ & $<20$ \\
\hline THIABENDAZOLE (1) & Anthelmintic & $<11$ & $<11$ & $<11$ & $<11$ & 4.5 \\
\hline Trimethoprim (1) & Antibiotic & $<14$ & $<14$ & $<14$ & $<14$ & $<14$ \\
\hline Trimethoprim (2) & Antibiotic & $<10$ & $<10$ & $<10$ & $<10$ & $<10$ \\
\hline Tylosin (2) & Antibiotic & $<20$ & $<20$ & $<20$ & $<20$ & $<20$ \\
\hline Virginiamycin (2) & Antibiotic & $<100$ & $<100$ & $<100$ & $<100$ & $<100$ \\
\hline Warfarin (1) & Anticoagulant & $<1$ & $<1$ & $<1$ & $<1$ & $<1$ \\
\hline
\end{tabular}

Numbers in parenthesis indicate the analytical method used.

Bold text indicates chemical residues found in POCIS.

Bold caps text indicates residues found only in the POCIS and not in the water-column samples.

Italics text indicates an estimated quantitation value (value is extrapolated below reporting level).

Methods: (1)—LC/MS; (2)—LC/MS; (3)—GC/MS.

chemicals of interest to pass through to the sorbent, while excluding particulate matter, biogenic material, and other large, potentially interfering substances. The polyethersulfone membrane (Pall Gelman Sciences, Ann Arbor, MI, USA) contains water-filled pores,
$0.1 \mu \mathrm{m}$ in diameter, to facilitate transport of the hydrophilic chemicals. The POCIS was designed to mimic respiratory exposure of aquatic organisms to dissolved chemicals without the inherent problems of metabolism, depuration of chemicals, avoidance of contaminated 
Table 2

Miscellaneous wastewater-related contaminants detected at Site 1

\begin{tabular}{|c|c|c|c|c|c|c|}
\hline Chemical (method) & Use & $\begin{array}{l}\text { Water } \\
\text { Rep. \#1 } \\
\text { ng/1 }\end{array}$ & $\begin{array}{l}\text { Water } \\
\text { Rep. \#2 } \\
\text { ng/1 }\end{array}$ & $\begin{array}{l}\text { Water } \\
\text { Rep. \#3 } \\
\text { ng/1 }\end{array}$ & $\begin{array}{l}\text { Water } \\
\text { Rep. \#4 } \\
\text { ng/l }\end{array}$ & $\begin{array}{l}\text { POCIS } \\
\text { ng/POCIS }\end{array}$ \\
\hline 1,7-Dimethylxanthine (1) & Caffeine metabolite & $<18$ & $<18$ & $<18$ & $<18$ & $<18$ \\
\hline 3,4 -Dichlorophenyl isocyanate (3) & Herbicide intermediate & 230 & 55 & 160 & 110 & $<500$ \\
\hline 3-METHYL-1H-INDOLE (3) & Odor in feces & NA & $<1000$ & $<1000$ & $<1000$ & 35 \\
\hline 3-tert-Butyl-4-hydroxyanisole (3) & Antioxidant & NA & $<5000$ & $<5000$ & $<5000$ & $<5000$ \\
\hline 4-CUMYLPHENOL (3) & Nonionic detergent metabolite & NA & $<1000$ & $<1000$ & $<1000$ & 80 \\
\hline 4-Octylphenol (3) & Nonionic detergent metabolite & NA & $<1000$ & $<1000$ & $<1000$ & $<1000$ \\
\hline 4-TERT-OCTYLPHENOL (3) & Nonionic detergent metabolite & NA & $<1000$ & $<1000$ & $<1000$ & 70 \\
\hline $\begin{array}{l}\text { 5-METHYL-1H- } \\
\text { BENZOTRIAZOLE (3) }\end{array}$ & Anticorrosive & NA & $<2000$ & $<2000$ & $<2000$ & 3600 \\
\hline Acetophenone (3) & Fragrance & NA & $<500$ & $<500$ & $<500$ & $<500$ \\
\hline Anthraquinone (3) & Manuf. dyes & NA & 73 & 36 & $<500$ & 30 \\
\hline Atrazine (3) & Herbicide & 91 & 110 & 15 & 200 & 300 \\
\hline Benzophenone (3) & Fixative in soaps/perfume & NA & 110 & 170 & 64 & 280 \\
\hline Bisphenol A (3) & Plasticizer & NA & 230 & $<1000$ & 98 & $<1000$ \\
\hline Bromacil (3) & Herbicide & NA & $<500$ & $<500$ & $<500$ & $<500$ \\
\hline Bromoform (3) & Ozination byproduct & NA & 71 & 20 & $<500$ & $<500$ \\
\hline Caffeine (1) & Stimulant & 21 & 29 & 55 & 37 & 2.7 \\
\hline Caffeine (3) & Stimulant & NA & $<500$ & 97 & 76 & 260 \\
\hline Camphor (3) & Antipruritic & NA & $<500$ & $<500$ & $<500$ & $<500$ \\
\hline Carbaryl (3) & Insecticide & NA & $<1000$ & $<1000$ & $<1000$ & $<1000$ \\
\hline Carbazole (3) & Manuf. dyes & NA & $<500$ & $<500$ & $<500$ & $<500$ \\
\hline Chlorpyrifos (3) & Insecticide & NA & $<500$ & $<500$ & $<500$ & $<500$ \\
\hline Cotinine (1) & Nicotine metabolite & 17 & 23 & 24 & 13 & 3.2 \\
\hline Cotinine (3) & Nicotine metabolite & NA & $<1000$ & 71 & $<1000$ & $<1000$ \\
\hline DEET ( $N, N$-diethyltoluamide) (3) & Insect repellant & NA & 99 & 82 & 51 & 320 \\
\hline DIAZINON (3) & Insecticide & NA & $<500$ & $<500$ & $<500$ & 65 \\
\hline 1,4-Dichlorobenzene (3) & Deodorizer & NA & $<500$ & $<500$ & $<500$ & $<500$ \\
\hline Dichlorvos (3) & Insecticide & NA & $<1000$ & $<1000$ & $<1000$ & $<1000$ \\
\hline Diethyl phthalate (3) & Plasticizer & $<500$ & $<500$ & $<500$ & $<500$ & $<500$ \\
\hline Diethylhexyl phthalate (3) & Plasticizer & 1900 & $<500$ & $<500$ & $<500$ & $<500$ \\
\hline $\begin{array}{l}\text { Fyrol CEF (3) (tri(2-chloroethyl) } \\
\text { phosphate) }\end{array}$ & Flame retardant & NA & 210 & 280 & 89 & 1000 \\
\hline $\begin{array}{l}\text { Fyrol FR2 (3) (tri(dichloroisopropyl) } \\
\text { phosphate) }\end{array}$ & Flame retardant & NA & 230 & 320 & 110 & 950 \\
\hline $\begin{array}{l}\text { Hexahydrohexamethylcyclo- } \\
\text { pentabenzopyran } \\
\text { (HHCB) (3) }\end{array}$ & Musk fragrance & NA & 170 & 320 & 120 & 1000 \\
\hline INDOLE (3) & Fragrance & NA & $<500$ & $<500$ & $<500$ & 230 \\
\hline Isoborneol (3) & Insecticide & NA & $<500$ & $<500$ & $<500$ & $<500$ \\
\hline Isophorone (3) & Solvent & NA & $<500$ & $<500$ & $<500$ & $<500$ \\
\hline Isopropyl benzene (cumene) (3) & Manuf. chemical & NA & $<500$ & $<500$ & $<500$ & $<500$ \\
\hline Isoquinoline (3) & Fragrance & NA & $<500$ & $<500$ & $<500$ & $<500$ \\
\hline d-Limonene (3) & Fungicide & NA & $<500$ & $<500$ & $<500$ & $<500$ \\
\hline Menthol (3) & Cough drops & NA & $<500$ & $<500$ & $<500$ & $<500$ \\
\hline Metalaxyl (3) & Fungicide & NA & $<500$ & $<500$ & $<500$ & $<500$ \\
\hline METHYL SALICYLATE (3) & Fragrance & NA & $<500$ & $<500$ & $<500$ & 65 \\
\hline Metolachlor (3) & Herbicide & NA & 35 & 17 & 54 & 130 \\
\hline$p$-cresol (3) & Preservative & NA & $<1000$ & $<1000$ & $<1000$ & $<1000$ \\
\hline PENTACHLOROPHENOL (3) & Insecticide & NA & $<2000$ & $<2000$ & $<2000$ & 130 \\
\hline Phenol (3) & Disinfectant & NA & $<500$ & $<500$ & $<500$ & $<500$ \\
\hline Prometon (3) & Herbicide & NA & $<500$ & 370 & $<500$ & 240 \\
\hline $\begin{array}{l}\text { NONYLPHENOL, DIETHOXY } \\
\text { (total NPEO2) (3) }\end{array}$ & Nonionic detergent metabolite & NA & $<5000$ & $<5000$ & $<5000$ & 1100 \\
\hline $\begin{array}{l}\text { Nonylphenol, monoethoxy } \\
\text { (total NPEO1) (3) }\end{array}$ & Nonionic detergent metabolite & NA & $<5000$ & $<5000$ & $<5000$ & $<5000$ \\
\hline
\end{tabular}


Table 2 (continued)

\begin{tabular}{|c|c|c|c|c|c|c|}
\hline Chemical (method) & Use & $\begin{array}{l}\text { Water } \\
\text { Rep. \#1 } \\
\text { ng/1 }\end{array}$ & $\begin{array}{l}\text { Water } \\
\text { Rep. \#2 } \\
\text { ng/1 }\end{array}$ & $\begin{array}{l}\text { Water } \\
\text { Rep. \#3 } \\
\text { ng/1 }\end{array}$ & $\begin{array}{l}\text { Water } \\
\text { Rep. \#4 } \\
\text { ng/1 }\end{array}$ & $\begin{array}{l}\text { POCIS } \\
\text { ng/POCIS }\end{array}$ \\
\hline 4-Nonylphenol (3) & Nonionic detergent metabolite & NA & $<5000$ & $<5000$ & $<5000$ & $<5000$ \\
\hline $\begin{array}{l}\text { 4-Octylphenol monoethoxylate } \\
\text { (total OPEO1) (3) }\end{array}$ & Nonionic detergent metabolite & NA & $<1000$ & $<1000$ & $<1000$ & $<1000$ \\
\hline $\begin{array}{l}\text { 4-Octylphenol diethoxylate } \\
\text { (total OPEO2) (3) }\end{array}$ & Nonionic detergent metabolite & NA & $<1000$ & $<1000$ & $<1000$ & $<1000$ \\
\hline Tetrachloroethylene (3) & Degreaser & NA & $<500$ & $<500$ & $<500$ & $<500$ \\
\hline Tonalide (AHTN) (3) & Musk fragrance & NA & 630 & 1100 & 430 & 3100 \\
\hline Tributyl phosphate (3) & Antifoaming & NA & 110 & 260 & $<500$ & 300 \\
\hline Triclosan (3) & Antimicrobial disinfectant & NA & 120 & 61 & 28 & 150 \\
\hline Triethyl citrate (3) & Cosmetics & NA & $<500$ & 78 & $<500$ & 170 \\
\hline Triphenyl phophate (3) & Plasticizer & NA & 54 & 12 & 66 & 35 \\
\hline Tri(2-butoxyethyl)phosphate (3) & Flame retardant & NA & $<500$ & 460 & 88 & 80 \\
\hline
\end{tabular}

Numbers in parenthesis indicate the analytical method used.

Bold text indicates chemical residues found in POCIS.

Bold caps text indicates residues found only in the POCIS and not in the water-column samples.

Italics text indicates an estimated quantitation value (value is extrapolated below reporting level).

NA - not analyzed, analysis for this chemical was not performed.

Methods: (1)—LC/MS; (2)—LC/MS; (3)—GC/MS.

areas, and mortalities of test organisms. Also, dietary uptake of polar organic compounds likely represents only a small fraction of residues accumulated in aquatic organism tissues (Huckins et al., 1997). Thus, the POCIS provides a worst case exposure scenario for aquatic organisms, enables concentration of sufficient amounts of bioavailable hydrophilic organic chemicals for some biomarker tests and permits determination of the biologically relevant TWA concentrations in water.

\section{Site selection and sampling}

Assunpink Creek in the vicinity of Trenton, New Jersey was selected for study (Fig. 2). This watershed is predominantly agricultural in its headwaters and becomes heavily urbanized in its lower reaches. A major municipal wastewater treatment plant (WWTP) is located near the center of the watershed serving greater than 100000 people. The WWTP discharges 10-12 million gallons per day of tertiary treated effluent into the creek. Assunpink creek is a tributary to the Delaware River, which is used further downstream as a source of drinking water for the city of Philadelphia and surrounding metropolitan areas. Two sites along the creek were selected to determine the presence and potential transport of organic contaminants. Site 1 is approximately 100 yards downstream from where the WWTP effluent is discharged and Site 2 is approximately 2 miles further downstream.

At each site, a protective canister containing eight POCIS devices, each with approximately $41 \mathrm{~cm}^{2}$ of effec- tive sampling surface area, was deployed for 54 days. The POCIS used conformed to the standard configuration of $180 \mathrm{~cm}^{2}$ sampling surface area per gram of sorbent (Alvarez et al., 2004). Of the eight POCIS devices per canister, four each of the generic configuration and the pharmaceutical configuration were used. Quality control (QC) measures included fabrication blanks and field blanks $(n=2)$ for each analytical technique. Fabrication blanks account for any background contribution due to interferences from POCIS components and for contamination incurred during laboratory storage, processing, and analytical procedures. Field blank POCIS are used as QC samples for transport, deployment and retrieval procedures (note that these POCIS are sealed back in the same shipping cans and stored frozen during the exposure period). The field blank POCIS are treated identically as the deployed devices, with the exception that they are not exposed to waters at the study sites.

Four stream samples were collected using standard depth and width water-column techniques (Shelton, 1994), at about 14 day intervals throughout the POCIS deployment period, and water-quality sampling field protocols (US Geological Survey, 1998). At each site, a composite sample of water was collected from about six vertical profiles and then split into duplicate baked, 1-liter (1) amber glass bottles. Samples requiring filtration were filtered through a $142 \mathrm{~mm}$ diameter, $0.7 \mu \mathrm{m}$ pore size, pre-baked, glass-fiber filter. All samples were immediately chilled and shipped overnight to participating laboratories. Quality control measures included the collection of blank water samples 
Table 3

Prescription and nonprescription pharmaceuticals detected at Site 2

\begin{tabular}{|c|c|c|c|c|c|c|}
\hline Chemical (method) & Use & $\begin{array}{l}\text { Water } \\
\text { Rep. \#1 } \\
\text { ng/l }\end{array}$ & $\begin{array}{l}\text { Water } \\
\text { Rep. \#2 } \\
\text { ng/1 }\end{array}$ & $\begin{array}{l}\text { Water } \\
\text { Rep. \#3 } \\
\text { ng/1 }\end{array}$ & $\begin{array}{l}\text { Water } \\
\text { Rep. \#4 } \\
\text { ng/1 }\end{array}$ & $\begin{array}{l}\text { POCIS } \\
\text { ng/POCIS }\end{array}$ \\
\hline Acetaminophen (1) & Analgesic & 14 & 13 & 31 & $<9$ & $<9$ \\
\hline Albuterol (salbutamol) (1) & Antiasthmatic & $<29$ & $<29$ & $<29$ & $<29$ & $<29$ \\
\hline Carbadox (2) & Antibiotic & $<50$ & $<50$ & $<50$ & $<50$ & $<50$ \\
\hline Carbamazepine (1) & Anticonvulsive & 64 & 54 & 90 & 16 & 150 \\
\hline Chlortetracycline (2) & Antibiotic & $<20$ & $<20$ & $<20$ & $<20$ & $<20$ \\
\hline Cimetidine (1) & Antacid & $<7$ & $<7$ & $<7$ & $<7$ & $<7$ \\
\hline Ciprofloxacin (2) & Antibiotic & $<10$ & $<10$ & $<10$ & $<10$ & $<10$ \\
\hline Codeine (1) & Analgesic & $<240$ & $<240$ & $<240$ & $<240$ & $<240$ \\
\hline Dehydronifedipine (1) & Antianginal & 5.5 & 6.9 & 13 & 1.2 & 14 \\
\hline Demeclocycline (2) & Antibiotic & $<20$ & $<20$ & $<20$ & $<20$ & $<20$ \\
\hline Diltiazem (1) & Antihypertensive & 11 & $<12$ & $<12$ & $<12$ & $<12$ \\
\hline DIPHENHYDRAMINE (1) & Antihistaminic & $<15$ & $<15$ & $<15$ & $<15$ & 8.2 \\
\hline Doxycycline (2) & Antibiotic & $<50$ & $<50$ & $<50$ & $<50$ & $<50$ \\
\hline Enrofloxacin (2) & Antibiotic & $<10$ & $<10$ & $<10$ & $<10$ & $<10$ \\
\hline Erythromycin- $\mathrm{H}_{2} \mathrm{O}(2)$ & Erythromycin metabolite & $<20$ & $<20$ & $<20$ & $<20$ & $<20$ \\
\hline Fluoxetine (1) & Antidepressant & $<18$ & $<18$ & $<18$ & $<18$ & $<18$ \\
\hline Furosemide (1) & Diuretic & $<39$ & $<39$ & $<39$ & $<39$ & $<39$ \\
\hline Gemfibrozil (1) & Antihyperlipidemic & $<15$ & $<15$ & $<15$ & $<15$ & $<15$ \\
\hline Ibuprofen (1) & Analgesic & $<18$ & $<18$ & $<18$ & $<18$ & $<18$ \\
\hline Lincomycin (2) & Antibiotic & $<10$ & $<10$ & $<10$ & $<10$ & $<10$ \\
\hline Methotrexate (2) & Antirheumatic & $<20$ & $<20$ & $<20$ & $<20$ & $<20$ \\
\hline Miconazole (1) & Antifungal & $<18$ & $<18$ & $<18$ & $<18$ & $<18$ \\
\hline Minocycline (2) & Antibiotic & $<20$ & $<20$ & $<20$ & $<20$ & $<20$ \\
\hline Norfloxacin (2) & Antibiotic & $<10$ & $<10$ & $<10$ & $<10$ & $<10$ \\
\hline Oxytetracycline (2) & Antibiotic & $<50$ & $<50$ & $<50$ & $<50$ & $<50$ \\
\hline Ranitidine (1) & Antacid & $<10$ & $<10$ & $<10$ & $<10$ & $<10$ \\
\hline Roxithromycin (2) & Antibiotic & $<10$ & $<10$ & $<10$ & $<10$ & $<10$ \\
\hline Sarafloxacin (2) & Antibiotic & $<10$ & $<10$ & $<10$ & $<10$ & $<10$ \\
\hline Sulfachlorpyradazine (2) & Antibiotic & $<50$ & $<50$ & $<50$ & $<50$ & $<50$ \\
\hline Sulfadimethoxine (2) & Antibiotic & $<10$ & $<10$ & $<10$ & $<10$ & $<10$ \\
\hline Sulfamerazine (2) & Antibiotic & $<20$ & $<20$ & $<20$ & $<20$ & $<20$ \\
\hline Sulfamethazine (2) & Antibiotic & $<10$ & $<10$ & $<10$ & $<10$ & $<10$ \\
\hline Sulfamethoxazole (1) & Antibiotic & 22 & $<23$ & 11 & $<23$ & 11 \\
\hline Sulfamethoxazole (2) & Antibiotic & $<50$ & $<50$ & $<50$ & $<50$ & $<50$ \\
\hline Sulfathiazole (2) & Antibiotic & $<50$ & $<50$ & $<50$ & $<50$ & $<50$ \\
\hline Tetracycline (2) & Antibiotic & $<50$ & $<50$ & $<50$ & $<50$ & $<20$ \\
\hline THIABENDAZOLE (1) & Anthelmintic & $<11$ & $<11$ & $<11$ & $<11$ & 15 \\
\hline Trimethoprim (1) & Antibiotic & $<14$ & $<14$ & $<14$ & $<14$ & $<14$ \\
\hline Trimethoprim (2) & Antibiotic & $<10$ & $<10$ & $<10$ & $<10$ & $<10$ \\
\hline Tylosin (2) & Antibiotic & $<20$ & $<20$ & $<20$ & $<20$ & $<20$ \\
\hline Virginiamycin (2) & Antibiotic & $<100$ & $<100$ & $<100$ & $<100$ & $<100$ \\
\hline Warfarin (1) & Anticoagulant & $<1$ & $<1$ & $<1$ & $<1$ & $<1$ \\
\hline
\end{tabular}

Numbers in parenthesis indicate the analytical method used.

Bold text indicates chemical residues found in POCIS.

Bold caps text indicates residues found only in the POCIS and not in the water-column samples.

Italics text indicates an estimated quantitation value (value is extrapolated below reporting level).

Methods: (1)—LC/MS; (2)— LC/MS; (3)—GC/MS.

derived from laboratory-grade or organic-free water to determine if sampling procedures, sampling equipment, field conditions, sample shipment and storage (field blank), or laboratory procedures (laboratory blank) introduced target analytes into environmental samples.

\section{Analytical methods}

\subsection{Recovery of chemical residues from POCIS}

Procedures for the recovery of the sequestered chemical residues from the deployed and QC POCIS are 
Table 4

Miscellaneous wastewater-related contaminants detected at Site 2

\begin{tabular}{|c|c|c|c|c|c|c|}
\hline Chemical (method) & Use & $\begin{array}{l}\text { Water } \\
\text { Rep. \#1 } \\
\text { ng/l }\end{array}$ & $\begin{array}{l}\text { Water } \\
\text { Rep. \#2 } \\
\text { ng/l }\end{array}$ & $\begin{array}{l}\text { Water } \\
\text { Rep. \#3 } \\
\text { ng/l }\end{array}$ & $\begin{array}{l}\text { Water } \\
\text { Rep. \#4 } \\
\text { ng/1 }\end{array}$ & $\begin{array}{l}\text { POCIS } \\
\text { ng/POCIS }\end{array}$ \\
\hline 1,7-Dimethylxanthine (1) & Caffeine metabolite & $<18$ & $<18$ & $<18$ & $<18$ & $<18$ \\
\hline 3,4-Dichlorophenyl isocyanate (3) & Herbicide intermediate & 90 & 47 & 42 & 55 & $<500$ \\
\hline 3-Methyl-1H-indole (3) & Odor in feces & NA & $<1000$ & $<1000$ & $<1000$ & $<1000$ \\
\hline 3-tert-Butyl-4-hydroxyanisole (3) & Antioxidant & NA & $<5000$ & $<5000$ & $<5000$ & $<5000$ \\
\hline 4-CUMYLPHENOL (3) & Nonionic detergent metabolite & NA & $<1000$ & $<1000$ & $<1000$ & 35 \\
\hline 4-Octylphenol (3) & Nonionic detergent metabolite & NA & $<1000$ & $<1000$ & $<1000$ & $<1000$ \\
\hline 4-TERT-OCTYLPHENOL (3) & Nonionic detergent metabolite & NA & $<1000$ & $<1000$ & $<1000$ & 70 \\
\hline 5-METHYL-1H-BENZOTRIAZOLE (3) & Anticorrosive & NA & $<2000$ & $<2000$ & $<2000$ & 2200 \\
\hline Acetophenone (3) & Fragrance & NA & $<500$ & $<500$ & $<500$ & $<500$ \\
\hline Anthraquinone (3) & Manuf. dyes & NA & 80 & 50 & $<500$ & 30 \\
\hline Atrazine (3) & Herbicide & 97 & 130 & 17 & 190 & 280 \\
\hline Benzophenone (3) & Fixative in soaps/perfume & NA & 130 & 110 & 31 & 230 \\
\hline Bisphenol A (3) & Plasticizer & NA & $<1000$ & $<1000$ & 400 & $<1000$ \\
\hline Bromacil (3) & Herbicide & NA & $<500$ & 62 & $<500$ & $<500$ \\
\hline Bromoform (3) & Ozination byproduct & NA & 27 & 77 & 35 & $<500$ \\
\hline Caffeine (1) & Stimulant & 53 & 45 & 81 & 17 & 23 \\
\hline Caffeine (3) & Stimulant & NA & $<500$ & 100 & 27 & 280 \\
\hline Camphor (3) & Antipruritic & NA & $<500$ & $<500$ & $<500$ & $<500$ \\
\hline Carbaryl (3) & Insecticide & NA & $<1000$ & $<1000$ & $<1000$ & $<1000$ \\
\hline Carbazole (3) & Manuf. dyes & NA & $<500$ & $<500$ & $<500$ & $<500$ \\
\hline Chlorpyrifos (3) & Insecticide & NA & $<500$ & $<500$ & $<500$ & $<500$ \\
\hline Cotinine (1) & Nicotine metabolite & 23 & 23 & 31 & 9.9 & 4.9 \\
\hline Cotinine (3) & Nicotine metabolite & NA & $<1000$ & 77 & $<1000$ & $<1000$ \\
\hline DEET ( $N, N$-diethyltoluamide) (3) & Insect repellant & NA & 340 & 82 & 45 & 240 \\
\hline DIAZINON (3) & Insecticide & NA & $<500$ & $<500$ & $<500$ & 65 \\
\hline 1,4-Dichlorobenzene (3) & Deodorizer & NA & $<500$ & $<500$ & $<500$ & $<500$ \\
\hline Dichlorvos (3) & Insecticide & NA & $<1000$ & $<1000$ & $<1000$ & $<1000$ \\
\hline Diethyl phthalate (3) & Plasticizer & NA & $<500$ & $<500$ & $<500$ & $<500$ \\
\hline DIETHYLHEXYL PHTHALATE (3) & Plasticizer & NA & $<500$ & $<500$ & $<500$ & 15 \\
\hline $\begin{array}{l}\text { Fyrol CEF (3) (tri(2-chloroethyl) } \\
\text { phosphate) }\end{array}$ & Flame retardant & NA & 310 & 190 & 36 & 600 \\
\hline $\begin{array}{l}\text { Fyrol FR2 (3) (tri(dichloroisopropyl) } \\
\text { phosphate) }\end{array}$ & Flame retardant & NA & 170 & 250 & 54 & 500 \\
\hline $\begin{array}{l}\text { Hexahydrohexamethylcyclopentabenzopyran } \\
\text { (HHCB) (3) }\end{array}$ & Musk fragrance & NA & 94 & 120 & 40 & 400 \\
\hline INDOLE (3) & Fragrance & NA & $<500$ & $<500$ & $<500$ & 440 \\
\hline Isoborneol (3) & Insecticide & NA & $<500$ & $<500$ & $<500$ & $<500$ \\
\hline Isophorone (3) & Solvent & NA & $<500$ & $<500$ & $<500$ & $<500$ \\
\hline Isopropyl benzene (cumene) (3) & Manuf. chemical & NA & $<500$ & $<500$ & $<500$ & $<500$ \\
\hline Isoquinoline (3) & Fragrance & NA & $<500$ & $<500$ & $<500$ & $<500$ \\
\hline d-Limonene (3) & Fungicide & NA & $<500$ & $<500$ & $<500$ & $<500$ \\
\hline Menthol (3) & Cough drops & NA & $<500$ & $<500$ & $<500$ & $<500$ \\
\hline Metalaxyl (3) & Fungicide & NA & $<500$ & $<500$ & $<500$ & $<500$ \\
\hline Methyl salicylate (3) & Fragrance & NA & 41 & $<500$ & $<500$ & 110 \\
\hline Metolachlor (3) & Herbicide & NA & 34 & 13 & 53 & 120 \\
\hline$p$-cresol (3) & Preservative & NA & 37 & $<1000$ & $<1000$ & $<1000$ \\
\hline PENTACHLOROPHENOL (3) & Insecticide & NA & $<2000$ & $<2000$ & $<2000$ & 130 \\
\hline Phenol (3) & Disinfectant & NA & 1100 & $<500$ & 500 & $<500$ \\
\hline Prometon (3) & Herbicide & NA & $<500$ & 340 & $<500$ & 210 \\
\hline Nonylphenol, diethoxy (total NPEO2) (3) & Nonionic detergent metabolite & NA & $<5000$ & $<5000$ & 410 & 850 \\
\hline $\begin{array}{l}\text { Nonylphenol, monoethoxy } \\
\text { (total NPEO1) (3) }\end{array}$ & Nonionic detergent metabolite & NA & $<5000$ & $<5000$ & $<5000$ & $<5000$ \\
\hline 4-Nonylphenol (3) & Nonionic detergent metabolite & NA & $<5000$ & $<5000$ & $<5000$ & $<5000$ \\
\hline 4-Octylphenol monoethoxylate & Nonionic detergent metabolite & NA & 1300 & $<1000$ & $<1000$ & $<1000$ \\
\hline
\end{tabular}


Table 4 (continued)

\begin{tabular}{|c|c|c|c|c|c|c|}
\hline Chemical (method) & Use & $\begin{array}{l}\text { Water } \\
\text { Rep. \#1 } \\
\text { ng/l }\end{array}$ & $\begin{array}{l}\text { Water } \\
\text { Rep. \#2 } \\
\text { ng/l }\end{array}$ & $\begin{array}{l}\text { Water } \\
\text { Rep. \#3 } \\
\text { ng/1 }\end{array}$ & $\begin{array}{l}\text { Water } \\
\text { Rep. \#4 } \\
\text { ng/l }\end{array}$ & $\begin{array}{l}\text { POCIS } \\
\text { ng/POCIS }\end{array}$ \\
\hline $\begin{array}{l}\text { 4-Octylphenol diethoxylate } \\
\text { (total OPEO2) (3) }\end{array}$ & Nonionic detergent metabolite & NA & $<1000$ & $<1000$ & $<1000$ & $<1000$ \\
\hline Tetrachloroethylene (3) & Degreaser & NA & $<500$ & $<500$ & $<500$ & $<500$ \\
\hline Tonalide (AHTN) (3) & Musk fragrance & NA & 340 & 500 & 150 & 1800 \\
\hline Tributyl phosphate (3) & Antifoaming & NA & 160 & 310 & $<500$ & 200 \\
\hline Triclosan (3) & Antimicrobial disinfectant & NA & 100 & 48 & $<1000$ & 100 \\
\hline Triethyl citrate (3) & Cosmetics & NA & $<500$ & 66 & $<500$ & 55 \\
\hline Triphenyl phophate (3) & Plasticizer & NA & 60 & 13 & 5.2 & 35 \\
\hline Tri(2-butoxyethyl)phosphate (3) & Flame retardant & NA & $<500$ & 170 & 110 & 90 \\
\hline
\end{tabular}

Numbers in parenthesis indicate the analytical method used.

Bold text indicates chemical residues found in POCIS.

Bold caps text indicates residues found only in the POCIS and not in the water-column samples.

Italics text indicates an estimated quantitation value (value is extrapolated below reporting level).

NA - not analyzed, analysis for this chemical was not performed.

Methods: (1)—LC/MS; (2)—LC/MS; (3)—GC/MS.

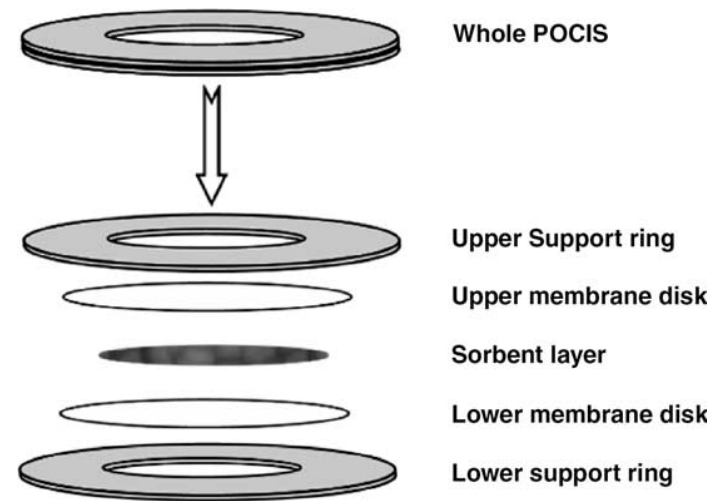

Fig. 1. Exploded view of a POCIS showing the sorbent layer contained between two membrane disks sandwiched between two support rings. A standard field deployed POCIS has an effective sampling surface area of $41 \mathrm{~cm}^{2}$.

described in detail by Alvarez et al. (2004). Briefly, the POCIS were disassembled and the sorbent was transferred into glass gravity-flow chromatography columns. Chemical residues were recovered from the sorbent by organic solvent elution. Methanol was used to recover the pharmaceuticals and a combination of 1:1:8 (v:v:v) methanol:toluene:dichloromethane was used for the other hydrophilic organic contaminants. All organic solvents were of Fisher Optima Grade or equivalent. The extracts were reduced in volume by rotary evaporation and under a gentle stream of nitrogen, filtered through glass-fiber filter, solvent exchanged to methanol as necessary, and ampoulated under nitrogen for shipment to the collaborating analytical laboratories. Each ampoulated sample was a composite of two individual POCIS extracts from the same deployment canister. These com- posites were created to increase the total mass of sequestered residues lowering analytical detection limits.

\subsection{Recovery of chemical residues from water-column samples}

The methods for water sample analysis are described by Kolpin et al. (2002). In general, the pharmaceuticals (Method 1) were extracted from 500 to $1000 \mathrm{ml}$ filtered water samples using Oasis HLB SPE cartridges (Waters, Milford, MA, USA) with methanol elution followed by methanol acidified with trichloroacetic acid. Method 2 for the antibiotics used a Waters mixed mode HLB-cation exchange (MCX) cartridge with subsequent elution by methanol with $5 \%$ ammonium hydroxide. Recovery of the remaining wastewater contaminants (Method 3) entailed the continuous liquid-liquid extraction (CLLE) with dichloromethane of 1-1 unfiltered whole water samples. The CLLE was performed for $3 \mathrm{~h}$ at ambient $\mathrm{pH}$ and for an additional $3 \mathrm{~h}$ at $\mathrm{pH} 2$. The extracts for each method were reduced in volume and transferred into the appropriate solvent prior to analysis.

\subsection{LC/MS analysis for pharmaceuticals in wastewater (Method 1)}

LC/MS was applied to aliquots of the extracts from the water samples and the POCIS pharmaceutical configuration using a Hewlett Packard (now Agilent Technologies, Inc., Palo Alto, CA, USA) Series 1100 HPLC/ MSD. The specifics of the LC/MS separation and analysis are reported by Cahill et al. (2004). An ammonium formate/formic acid buffer ( $10 \mathrm{mM}, \mathrm{pH} 3.7)$ aqueous phase and acetonitrile were used to produce a multistep binary elution gradient. The flow rate was 0.200 


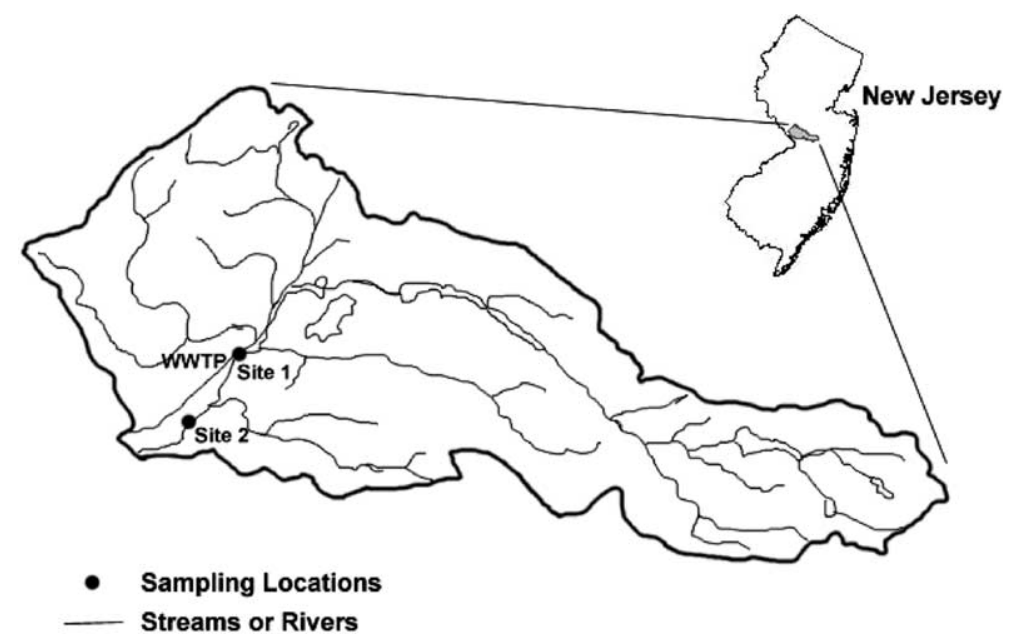

Fig. 2. Map of the sampling sites in the Assunpink Creek watershed in New Jersey, USA. Assunpink Creek is a tributary to the Delaware River which flows along the western border of the state. Site 1 and Site 2 are approximately two miles apart.

$\mathrm{ml} / \mathrm{min}$, and all flow was directed to the mass spectrometer. Separations were made using a Metasil Basic $3 \mu \mathrm{m}$, $150 \mathrm{~mm} \times 2.0 \mathrm{~mm}, \mathrm{C}-18$ analytical column coupled to either a Metasil Basic Safeguard (MetaChem Technologies, Lake Forest, CA, USA), $3 \mu \mathrm{m}, 2.0-\mathrm{mm}$ guard column, or NewGuard RP-18, $7 \mu \mathrm{m}, 15 \mathrm{~mm} \times 3.2 \mathrm{~mm}$ guard column (Perkin Elmer, Torrance, CA, USA).

The HPLC was interfaced with the mass spectrometer using electrospray ionization (ESI) in the positive ionization mode. The ESI source conditions were as follows: source temperature $150^{\circ} \mathrm{C}$, nebulizer gas pressure of $100 \mathrm{kPa}$, drying gas (nitrogen) flow rate of $91 / \mathrm{min}$, and drying gas temperature of $350^{\circ} \mathrm{C}$. The potential difference between the source and the capillary was held at 3500 V. Programmed capillary exit voltage changes were used to produce sufficient fragmentation of each compound so that characteristic fragments were produced. For each compound, the optimal detection conditions for the protonated molecular ion and at least one confirming fragment ion were used when collecting data in the selected ion monitoring (SIM) mode, thereby increasing the sensitivity of detection. A multipoint internal standard calibration, from 0.010 to $2.0 \mu \mathrm{g} / \mathrm{l}$, was used for each sample set analyzed.

\subsection{LC/MS analysis for antibiotics in wastewater (Method 2)}

Aliquots of the sample extracts from the water samples and the POCIS pharmaceutical configuration were concentrated ten fold by reducing $1 \mathrm{ml}$ sample aliquots to $20 \mu \mathrm{l}$ using nitrogen evaporation to which $80 \mu \mathrm{l}$ of $20 \mathrm{mM}$ ammonia acetate buffered water (pH 5.6) was added. Samples were analyzed using liquid chromatography/electrospray-mass spectrometry (LC/ESI-MS) in positive-ion mode using SIM using a Waters 1096 LC with a ZQ MS (Waters, Milford, MA, USA). The sulfonamide and macrolide classes of antibiotics along with lincomycin, trimethoprim, and carbadox were analyzed using a gradient separation described by Hirsch et al. (1998) with a Luna $3.0 \times 150 \mathrm{~mm}, 3.5 \mu \mathrm{m}$ phenylhexyl column (Phenomonex, Torrance, CA, USA). The tetracycline and fluoroquinolone compounds were analyzed using a gradient separation (Lindsey et al., 2001) with a Luna $3.0 \times 150 \mathrm{~mm}, 3.0 \mu \mathrm{m} \mathrm{C}_{8}(2)$ (Phenonomonex) or $3.0 \times 150 \mathrm{~mm}, 3.5 \mu \mathrm{m} \mathrm{C}_{18}$ MS Xterra (Waters, Milford, MA, USA) column. The LC conditions were: sample injection volume of $20 \mu \mathrm{l}$, flow rate of $0.3 \mathrm{ml} / \mathrm{min}$, autosampler temperature at $20^{\circ} \mathrm{C}$, and column heater temperature at $50^{\circ} \mathrm{C}$. The mass spectrometer conditions were: drying gas flow rate of $500 \mathrm{l} / \mathrm{h}$, cone gas flow rate of $50 \mathrm{l} / \mathrm{h}$, capillary voltage of $3.0 \mathrm{kV}$, source temperature at $100^{\circ} \mathrm{C}$, desolvation temperature at $220^{\circ} \mathrm{C}$, and low and high mass resolution of 15.0 .

\subsection{GC/MS analysis for miscellaneous wastewater contaminants (Method 3)}

The samples from the water samples and the generic POCIS configuration were analyzed by GC/MS using an Agilent 6890 GC (Agilent Technologies, Wilmington, DE, USA) with a 5973 mass selective detector (Agilent Technologies, Palo Alto, CA, USA) operated in electron impact, full scan mode, and equipped with a $25 \mathrm{~m} \times$ $0.2 \mathrm{~mm}, 0.33 \mu \mathrm{m}$ film thickness, $5 \%$ phenylmethylsilicone (Ultra II, Agilent) capillary column. The specific MS conditions are reported by Zaugg et al. (2002). The initial oven temperature was $40^{\circ} \mathrm{C}$, ramped at $9^{\circ} \mathrm{C} / \mathrm{min}$ to $300^{\circ} \mathrm{C}$, and held for $20 \mathrm{~min}$. The samples were analyzed in splitless mode with an injection 
temperature of $300{ }^{\circ} \mathrm{C}$ using $2 \mu$ injections. Quantitation was accomplished using internal standards and a 7-point calibration curve.

\section{Results and discussion}

Field and laboratory blanks were analyzed for target compounds during the course of this study. A field blank was prepared during the first stream sampling event and analyzed for target compounds by Methods 1-3. None of the target compounds in Methods 1 and 2 were detected in the field blank; phenol was detected by Method 3 at a concentration of $0.65 \mu \mathrm{g} / \mathrm{l}$. A subsequent field blank was prepared during the following stream sampling event and analyzed for target compounds in Method 3. Again, phenol was the only compound detected at a concentration of $0.41 \mu \mathrm{g} / \mathrm{l}$. Environmental concentrations of phenol for this investigation ranged from 0.23 to $0.66 \mu \mathrm{g} / \mathrm{l}$. Five laboratory blanks were analyzed for target compounds by Method 1. Acetaminophen was detected in one laboratory blank at $0.0033 \mu \mathrm{g} / \mathrm{l}$ but was not detected in associated environmental samples. Caffeine was detected in one laboratory blank at a concentration of $0.03 \mu \mathrm{g} / \mathrm{l}$. The detection of caffeine in an associated environmental sample less than this concentration was censored. Three laboratory set blanks were analyzed for target compounds by Method 3. 4-Octylphenol monoethoxylate (OPEO1), 4-tert-octylphenol, and para-nonylphenol were detected in one or more laboratory blanks but were not detected in any environmental samples for this study. Bisphenol A, tri(2-butoxyethyl)phosphate, and diethylhexyl phthalate (DEHP) were detected in one or more laboratory blanks but were not detected in any of the associated environmental samples. Phenol was detected in one set of laboratory blanks, however, the phenol data has already been censored due to its presence in the field blanks.

Quality control measures used with the POCIS included fabrication and field blanks which were prepared concomitantly with the field deployed POCIS. Processing and analysis of POCIS fabrication and field blanks were concurrent with and identical to that of the deployed POCIS. Analysis of the POCIS fabrication and field blanks resulted in the detection of only three targeted chemicals. The fabrication and field blank from Site 1 contained 30 and $120 \mathrm{ng} /$ POCIS, respectively, of 4-octylphenol diethoxylate (total OPEO2). Because the residues found in the field blank were larger than found in the Site 1 deployed sample, the data was censored. Residues of hexahydrohexamethylcyclopentabenzopyran (HHCB) were detected in the fabrication blanks (100 ng/POCIS) but not in the field blanks. The reported values for the deployed samples were fabrication blankbackground corrected. DEHP was found at levels in the field blank above the measured values in deployed
POCIS for Site 1 and was subsequently censored. Site 2 field blank levels of DEHP were less than that found in the deployed sample therefore the reported value was field blank-background corrected.

Residues from field deployed POCIS were compared to standard water-column samples taken concurrently at the same two sites in Assunpink Creek which receives agricultural, municipal, and industrial wastewaters. Water-column samples were taken at two week intervals during the 54 day POCIS deployment. Individual results from the four water-column samples taken and the POCIS deployed at each site are presented in Tables $1-4$.

Out of a total of 96 targeted analytes, 24 were identified in the water-column samples and 32 were identified in the POCIS extracts. Representative chemicals sampled by both techniques include pharmaceuticals (acetaminophen, carbamazepine, dehydronifedipine, diphenhydramine, and sulfamethoxazole), herbicides (atrazine, metolachlor, and prometon), flame retardants (Fyrol CEF and Fyrol FR2), and ingredients from personal care and consumer products (anthraquinone, benzophenone, caffeine, cotinine, DEET, HHCB, methyl salicylate, tonalide (AHTN), and triclosan). Ten chemicals were found only in the POCIS extracts which included diazinon, DEHP, indole, 5-methyl-1H-benzotriazole, pentachlorophenol, thiabendazole, and several alkyl phenols (nonionic detergent metabolites). The water-column samples contained six chemicals which were not detected in the POCIS extracts. These chemicals included diltiazem, 3,4-dichlorophenyl isocyanate, bisphenol A, bromoform, 4-octylphenol monoethoxylate (total OPEO1), and $p$-cresol. Concentrations of the chemicals detected only in the water-column samples were generally less than either the laboratory reporting level or the lowest calibration standard and thus are estimates which cannot be accurately quantitated (Childress et al., 1999).

Advantages of using integrative samplers providing TWA concentrations of contaminants is evidenced by the detection of chemicals which dissipate quickly or enter the watershed via an episodic event. This transient nature of many chemicals, especially the more water soluble ones, was observed by the erratic detections in the four water-column samples. Only 15 of the 26 chemicals detected in the water-column samples from Site 1 and 15 of 31 chemicals at Site 2 were present in each of the four water collections. Representative chemicals present in all the water samples included atrazine, caffeine, carbamazepine, dehydronifedipine, DEET, Fyrol CEF and FR2, metolachlor, and tonalide (Tables 1-4). Of the chemicals sporadically identified in the water-column samples, 8 of 11 were detected in POCIS extracts from Site 1 and 10 of 16 in POCIS extracts from Site 2. The data indicates that the POCIS is an effective substitute for an exhaustive sampling regiment to monitor for many transient chemicals. 
There were four cases where the presence of a chemical was examined by two separate analytical methods. The pharmaceuticals sulfamethoxazole and trimethoprim were determined using Methods 1 and 2. Sulfamethoxazole was detected in both the water-column and POCIS samples by Method 1, albeit very near the method detection limit, but not by Method 2. Neither method detected trimethoprim. These two antibiotics are widely used in tandem at a ratio of 5:1 sulfamethoxazole:trimethoprim for the treatment of urinary tract and middle ear infections, bronchitis, and pneumonia [www.fda.gov]. Due to the trace concentrations of sulfamethoxazole detected, it is likely that the concentration of trimethoprim may have fallen below the method detection limits. Additionally, trimethoprim may be more efficiently removed through tertiary wastewater treatment than sulfamethoxazole.

Caffeine, a commonly used marker for wastewater contamination (Seiler et al., 1999) was measured in both water-column samples and POCIS extracts by Methods 1 and 3. Cotinine, a nicotine metabolite, was detected in both the water-column samples and POCIS extracts by Method 1, but only in the water-column samples by Method 3. Due to the much higher detection limits for cotinine by Method 3, it is possible that residues were sequestered by the POCIS albeit at levels below the detection limit or the cotinine in the water-column samples was the result of contamination during sampling and/or analysis.

Differences in the results between the two analytical methods may be due to inherent variability of the methods, analytical sensitivity (i.e., detection limits) specific to each method, and variability in sample replicates. Each laboratory was sent an equivalent, although distinctly individual, sample for analysis. As stated earlier, the POCIS devices were tailored for two general classes of analytes. Laboratories using Methods 1 and 2 received extracts from POCIS tailored for pharmaceuticals and the laboratory using Method 3 received extracts from POCIS tailored for general hydrophilic organic contaminants. Although there is significant overlap in the sequestration of chemicals by both POCIS configurations, the final chemical recovery from the sorbents may be different. As this was a pilot study, method recoveries for the target chemicals from the POCIS were not determined.

Site to site variation in the number of chemicals detected and their associated concentrations was minimal. The levels of pharmaceuticals were lower, in some cases only slightly, at Site 2 than at Site 1. In some cases, the levels of OWCs were slightly higher at Site 2 than at Site 1 suggesting there may be another source for these chemicals other than the primary WWTP. This data tends to indicate that due to the higher water solubility of many of these chemicals and their apparent resistance to degradation, many OWCs are highly mobile and re- main in the water column over extended distances and periods of time.

Comparison of the sampling techniques was based on qualitative determinations (i.e., presence, or lack there of, of chemical). Data from the standard water-column sampling methods (Tables 1-4) are reported as nanogram of analyte per liter of water. The POCIS data (Tables 1-4) are reported as nanogram of analyte per single POCIS device. Estimation of the ambient water concentrations was not possible for the identified POCIS compounds due to a lack of calibration data. In order to estimate water concentrations from sequestered POCIS residues, the in situ sampling rates $\left(R_{\mathrm{S}}\right)$ for each chemical must be known. Such laboratory-derived $R_{\mathrm{S}}$ can be used in the following model describing integrative (i.e., linear) sampling to estimate the ambient water concentrations (Alvarez et al., 2004).

$C_{\mathrm{W}}=C_{\mathrm{S}} M_{\mathrm{S}} / R_{\mathrm{S}} t$

where $C_{\mathrm{W}}$ and $C_{\mathrm{S}}$ are the analyte concentration in the water and POCIS sorbent, respectively, $M_{\mathrm{S}}$ is the mass of the sorbent and $t$ is the time in days. Published $R_{\mathrm{S}}$ data for detected chemicals were not available. Laboratory investigations to provide calibration data for additional analytes are currently ongoing. In addition, an adaptation of the permeability/performance reference compound approach for the POCIS will be completed, which will provide a means of adjusting laboratoryderived calibration data for site-specific variables (turbulence, temperature, etc.) to increase the accuracy of estimated chemical water concentrations (Huckins et al., 2002b; Alvarez et al., 2004).

\section{Conclusions}

This comparison of traditional water-column sampling methodologies to a passive sampling technique for polar organic chemicals resulted in the most comprehensive list of chemical contaminants determined in POCIS sample extracts to date. Review of the data generated by both sampling methods indicates that the passive sampling method has advantages over traditional water-column sampling regimes. Eight additional chemicals were isolated from the POCIS extracts than from the water samples with twice as many found only in the POCIS compared to only in the water samples. Chemical residues found only in the POCIS were likely due to the TWA sequestration of trace levels of those chemicals from the water over the deployment period. These trace levels are often below the method detection limits obtainable with a sample of a few liters of water. The POCIS also samples chemicals that may enter the aquatic system via an episodic event and have a relatively short residence time (i.e., chemical/biological degradation, sorption, dissipation) which can be missed 
by taking instantaneous (water-column) samples as evidenced by the detection of chemicals by the POCIS which were present in only a few of the individual water-column samples. Optimization of the POCIS method for many of the chemical classes previously not investigated in our laboratory should lead to increased numbers of positive identifications. The use of passive samplers eliminates the need to perform multiple sampling operations to generate TWA concentrations of targeted chemicals. Generating multiple numbers of samples for the same amount of information provided by a single passive sampler is logistically and financially imprudent as part of a regular monitoring program. Passive samplers are typically easier to handle, transport and preserve than water samples comprising of one or more liters. Thus, the POCIS provides an increase in method sensitivity, simplicity in use, and relevance to ecological risk assessments not easily obtainable with traditional methods.

\section{References}

Alvarez, D.A., Petty, J.D., Huckins, J.N., Jones-Lepp, T.L., Getting, D.T., Goddard, J.P., Manahan, S.E., 2004. Development of a passive, in situ, integrative sampler for hydrophilic organic contaminants in aquatic environments. Environ. Toxicol. Chem. 23, 1640-1648.

American Conference of Governmental Industrial Hygienists (ACGIH), 1990. Threshold Limit Values for Chemical Substances and Physical Agents and Biological Exposure Indicies. ACGIH, Cincinnati, OH.

Barceló, D., Hennion, M., 1997. Sampling of polar pesticides from water matrices. Anal. Chim. Acta 338, 3-18.

Cahill, J.D., Furlong, E.T., Burkhardt, M.R., Kolpin, D.W., Anderson, L.R., 2004. Determination of pharmaceutical compounds in surface- and ground-water samples by solidphase extraction and high-performance liquid chromatography/electrospray-ionization mass spectrometry. J. Chromatogr. A 1041, 171-180.

Childress, C.J.O., Foreman, W.T., Connor, B.F., Maloney, T.J., 1999. New reporting procedures based on long-term method detection levels and some considerations for interpretations of water-quality data provided by the US Geological Survey National Water Quality Laboratory. US Geological Survey Open-File Report 99-193, Reston, VA.

Daughton, C., Ternes, T., 1999. Pharmaceuticals and personal care products in the environment: agents of subtle change? Environ. Health Perspect. 107, 907-938.

Desbrow, C., Routledge, E., Brighty, G., Sumpter, J., Waldock, M., 1998. Identification of estrogenic chemicals in stw effluent. 1. chemical fractionation and in vitro biological screening. Environ. Sci. Technol. 32, 1549-1558.

Fowler, W.K., 1982. Fundamentals of passive vapor sampling. Am. Lab. 14, 80-87.

Gustavson, K.E., Harkin, J.M., 2000. Comparison of sampling techniques and evaluation of semipermeable membrane devices (SPMDs) for monitoring polynuclear aromatic hydrocarbons (PAHs) in groundwater. Environ. Sci. Technol. 34, 4445-4451.

Halling-Sørensen, B., Nors Nielsen, S., Lanzley, P., Ingerslev, F., Holten Lützhøft, H., Jørgensen, S., 1998. Occurrence, fate and effects of pharmaceuticals substances in the environment-a review. Chemosphere 36, 357-393.

Hirsch, R., Ternes, T.A., Haberer, K., Mehlich, A., Ballwanz, F., Kratz, K.L., 1998. Determination of antibiotics in different water compartments via liquid chromatographyelectrospray tandem mass spectrometry. J. Chromatogr. A 815, 213-223.

Hilton, M.J., Thomas, K.V., 2003. Determination of selected human pharmaceutical compounds in effluent and surface water samples by high-performance liquid chromatography-electrospray tandem mass spectrometry. J. Chromatogr. A 1015, 129-141.

Huckins, J.N., Tubergen, M.W., Manuweera, G.K., 1990. Semipermeable membrane devices containing model lipid: a new approach to monitoring the availability of lipophilic contaminants and estimating their bioconcentration potential. Chemosphere 20, 533-552.

Huckins, J.N., Petty, J.D., Thomas, J., 1997. Bioaccumulation: how chemicals move from the water into fish and other aquatic organisms. American Petroleum Institute (API); API publication number 4656, Washington, DC, USA.

Huckins, J.N., Petty, J.D., Prest, H.F., Clark, R.C., Alvarez, D.A., Orazio, C.E., Lebo, J.A., Cranor, W.L., Johnson, B.T., 2002a. A guide for the use of semipermeable membrane devices (SPMDs) as samplers of waterborne hydrophobic organic contaminants. American Petroleum Institute (API); API publication number 4690, Washington, DC, USA.

Huckins, J.N., Petty, J.D., Lebo, J.A., Almeida, F.V., Booij, K., Alvarez, D.A., Cranor, W.C., Clark, R.C., Mogensen, B.B., 2002b. Development of the permeability/performance reference compounds approach for in situ calibration of semipermeable membrane devices. Environ. Sci. Technol. 26, 85-91.

Kolpin, D.W., Furlong, E.T., Meyer, M.T., Thurman, E.M., Zaugg, S.D., Barber, L.B., Buxton, H.T., 2002. Pharmaceuticals, hormones, and other organic wastewater contaminants in US streams, 1999-2000: a national reconnaissance. Environ. Sci. Technol. 36, 1202-1211.

Lebo, J.A., Gale, R.W., Petty, J.D., Tillitt, D.E., Huckins, J.N., Meadows, J.C., Orazio, C.E., Echols, K.R., Schroeder, D.J., Inmon, L.E., 1995. Use of the semipermeable membrane device as an in situ sampler of waterborne bioavailable PCDD and PCDF residues at sub-part-per-quadrillion concentrations. Environ. Sci. Technol. 29, 2886-2892.

Lindsey, L.E., Meyer, M.T., Thurman, E.M., 2001. Analysis of trace levels of sulfonamide and tetracycline antimicrobials in groundwater and surface water using solid-phase extractions and liquid chromatography/mass spectrometry. Anal. Chem. 74, 4640-4646.

Petty, J.D., Orazio, C.E., Huckins, J.N., Gale, R.W., Lebo, J.A., Meadows, J.C., Echols, K.R., Cranor, W.L., 2000. Considerations involved with the use of semipermeable membrane devices for monitoring environmental contaminants. J. Chromatogr. A 879, 83-95.

Petty, J.D., Huckins, J.N., Alvarez, D.A., 2002. Device for sequestration and concentration of polar organic chemicals from water. US Patent 6,478,961, November 12, 2002. 
Petty, J.D., Huckins, J.N., Alvarez, D.A., Brumbaugh, W.G., Cranor, W.L., Gale, R.W., Rastall, A.C., Jones-Lepp, T.L., Leiker, T.J., Rostad, C.E., Furlong, E.T., 2004. A holistic passive integrative sampling approach for assessing the presence and potential impacts of waterborne environmental contaminants. Chemosphere 54, 695-705.

Richardson, S., 2002. Environmental mass spectrometry: emerging contaminants and current issues. Anal. Chem. 74, 2719-2742.

Seiler, R.L., Zaugg, S.D., Thomas, J.M., Howcroft, D.L., 1999. Caffeine and pharmaceuticals as indicators of waste water contamination in wells. Ground Water 37, 405-410.

Shelton, L.R., 1994. Field guide for collecting and processing stream-water samples for the National Water Quality Assessment Program. Open-File Report number 94-455, US Geological Survey.
Stackelberg, P.E., Furlong, E.T., Meyer, M.T., Zaugg, S.D., Henderson, A.K., Reissman, D.B., 2004. Persistence of pharmaceutical compounds and other organic wastewater contaminants in a conventional drinking water treatment plant. Sci. Total Environ. 329, 99-113.

Steur-Lauridsen, F., 2003. Personal communication.

US Geological Survey, 1998. Techniques of water-resources investigations. Handbooks for Water-Resources Investigations, Book 9.

Zaugg, S.D., Smith, S.G., Schroeder, M.P., Barber, L.B., Burkhardt, M.R., 2002. Methods of analysis by the US Geological Survey National Water Quality Laboratory-Determination of wastewater compounds by polystyrene-divinylbenzene solid-phase extraction and capillary-column gas chromatography/mass spectrometry. Water-Resources Investigations Report 01-4186, US Geological Survey, Denver, CO. 